\title{
Belgeo
}

Revue belge de géographie

$4 \mid 2005$

Miscellaneous

\section{Développement durable et participation : la démocratie introuvable}

Sustainable development and participation: the unattainable democracy

\section{Romain Felli}

\section{(2) OpenEdition}

1 Journals

\section{Édition électronique}

URL : http://journals.openedition.org/belgeo/12126

DOI : 10.4000/belgeo.12126

ISSN : 2294-9135

Éditeur:

National Committee of Geography of Belgium, Société Royale Belge de Géographie

\section{Édition imprimée}

Date de publication : 31 décembre 2005

Pagination : 425-434

ISSN : 1377-2368

\section{Référence électronique}

Romain Felli, « Développement durable et participation : la démocratie introuvable », Belgeo [En ligne], 4 | 2005, mis en ligne le 29 octobre 2013, consulté le 10 décembre 2020. URL : http:// journals.openedition.org/belgeo/12126; DOI : https://doi.org/10.4000/belgeo.12126

Ce document a été généré automatiquement le 10 décembre 2020.

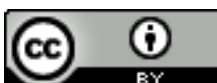

Belgeo est mis à disposition selon les termes de la licence Creative Commons Attribution 4.0 International. 


\section{Développement durable et participation : la démocratie introuvable}

Sustainable development and participation: the unattainable democracy

Romain Felli

\section{Introduction}

1 Si de nombreux travaux sont consacrés à la sociologie de la " démocratie participative ", un manque se fait encore sentir quant à des réflexions plus théoriques sur cet objet hybride. En particulier, un aspect mérite d'être souligné, celui de la coïncidence entre le "développement durable » et la problématique "participative». A l'heure où de plus en plus de villes se dotent d'un « agenda $21 »$ et entreprennent des démarches participatives avec leur population, une analyse des liens entre ces deux concepts mérite d'être faite.

2 Cet article tourne autour de deux questions principales, intrinsèquement liées. Premièrement, d'un point de vue analytique, il cherche à comprendre pourquoi le développement durable valorise l'idée de démocratie participative. Deuxièmement, d'un point de vue normatif, il se demande si cette nouvelle forme de démocratie est réellement plus démocratique que les alternatives qu'elle prétend remplacer (ou auxquelles elle viendrait s'ajouter).

3 Par démocratie nous entendrons la capacité du peuple (c'est-à-dire de n'importe qui, a priori) de prendre effectivement part à la décision politique. Cette égalité politique, c'est l' isonomia grecque (voir Vernant, 1962, p. 56 ; Manin, 1996, pp. 28-29). Evidemment, de nombreuses autres définitions du terme " démocratie » existent et sont en concurrence. Néanmoins celle-ci à l'avantage de dégager un critère strict permettant de mesurer l'écart entre l'idéal et le réel. Par ailleurs, la plupart des «effets » (réputés positifs ou non) de la démocratie, proviennent de cette égalité fondamentale (Finley, 1985, pp. 111-144; Rancière, 2005). Le raisonnement portera sur les institutions et les possibilités 
formelles de cette participation. Il existe par ailleurs de nombreuses études sur les modalités effectives de réalisation des procédures participatives portant notamment sur la sociologie de leurs utilisateurs (Bevort, 2002 ; Rui, 2004 ; Bacqué et al., 2005 ; Bourg et Boy, 2005). Néanmoins, un raisonnement formel peut mettre en évidence certains mécanismes qui permettent de répondre à nos questions.

Dans un premier temps, nous nous attacherons aux origines conceptuelles du développement durable, ce qui nous permettra, dans un second temps, de mieux comprendre le rapport ambigu que ce concept entretient à la démocratie. Puis, nous passerons à une réflexion formelle sur les différents types de démocratie, qui devrait montrer les limites du concept de "démocratie participative " tel qu'entendu par le développement durable. Nous pourrons alors conclure sur le développement durable comme tentative non-dialectique de concilier les contraires.

5 L'esprit du temps est au développement durable. Dans un élan quasi-unanime, toutes les entités politiques, de l'échelon du quartier à celui des agences internationales, toute la société civile, apportent leur pierre à ce grand oeuvre. Les sciences sociales, la géographie et la science politique en particulier, sont sommées de concourir également à ce but. Pourtant, avant d'être au service du prince (celui-ci fût-il le peuple dans son ensemble), les sciences sociales supposent une exigence critique. C'est cette exigence que nous aimerions porter ici.

\section{Le développement durable contre l'écologie politique}

Contrairement à une représentation courante, le développement durable doit être pensé comme s'étant construit conceptuellement contre l'écologie politique. Montrer précisément la manière dont s'organise cette opposition nécessiterait une recherche en soi, mais en en exposant les grandes lignes, nous devrions arriver à une meilleure intelligence de ce qu'est le développement durable, et, partant, de sa conception de la démocratie.

7 Cet article s'appuie sur la définition du développement durable issue du rapport Brundtland datant de 1987. En se basant sur ce document, sur les rapports subséquents de l'ONU (à Rio et Johannesburg notamment) ainsi que les textes de l'OCDE (2001 et 2002), une conception assez précise de ce qu'est le développement durable sera exposée. Certes, d'autres conceptions de ce concept sont ainsi écartées, néanmoins il faut admettre que c'est la conception «Brundtland» qui est la plus répandue tant chez les praticiens (politiques, experts...) que chez les académiques, ce qui justifie amplement cette réduction (Zaccaï, 2002 ; Jakubec, 2004 ; Smouts, 2005).

Quant à l'écologie politique, si ce courant est assez divers, certains auteurs apparaissent comme idéaux-typiques de la conception que nous étudions: Ivan Illich, André Gorz, Cornelius Castoriadis ou René Dumont notamment (pour plus de détails sur ce choix, voir Bourg, 1996; Alphandéry et al., 1991, p.162). Cette écologie politique, forte dans les années 1970, subsiste aujourd'hui chez certaines fractions des partis verts ou chez les tenants de la décroissance, comme par exemple José Bové. C'est sur les textes des auteurs susmentionnés que nous nous appuierons pour construire l'opposition conceptuelle entre développement durable et écologie politique.

A la base de ces deux doctrines se trouvent deux concepts fondamentaux et différents. Le développement durable est une pensée de la survie (Brundtland, 1987, p.1 et p.33), alors 
que l'écologie politique est une pensée de l'autonomie (Cohn-Bendit et Castoriadis, 1981). De cette différence fondamentale découlent toute une série d'autres oppositions dont quelques unes des plus importantes sont maintenant esquissées.

Tout d'abord sur le plan économique : l'écologie politique est une critique radicale de la société industrielle, et du mode de production capitaliste en particulier (le socialisme centralisé et productiviste est également la cible de ses attaques). Le capitalisme pose problème tant du point de vue des inégalités qu'il suppose que du « schème d'imposition des besoins » auquel il soumet les individus (cf. Cohn-Bendit et Castoriadis, 1981, pp. 36-37). Le développement durable, a contrario, ne remet en cause ni le capitalisme, ni le système productiviste. Il cherche par contre à « réorienter » l'économie afin de la rendre plus durable. Il suppose donc que des solutions techniques pourront être apportées aux problèmes (notamment environnementaux) issus de la production économique. Certains de ses tenants vont même jusqu'à affirmer que «le développement durable n'est ni une utopie ni même une contestation, mais la condition de survie de l'économie de marché ${ }^{1}$ ».

11 Deuxièmement, l'écologie politique est une critique radicale de l'Etat et de l'appareil étatique. Dans la lignée de la deuxième gauche, à laquelle elle peut être rattachée par certains aspects, l'écologie politique oppose à l'hétéronomie de l'Etat les valeurs de la société civile. L'Etat apparaît alors comme le fer de lance de l'économie industrielle et productiviste, et ainsi le premier obstacle à la société « conviviale ». Bien au contraire, le développement durable est une écologie de, par et pour l'Etat. Issu de cercles étatiques, inter-gouvernementaux, il fonde son action sur l'action de l'Etat et se conçoit toujours comme une politique publique (au sens de policy) à mettre en oeuvre.

Troisièmement, l'écologie politique est une critique radicale de la technique. D'après elle, le développement de la technologie conduit nécessairement au développement de la société industrielle, spécialisée, productiviste et hétéronome. Le développement durable, au contraire, adopte une attitude "neutre » vis-à-vis de la technique; celle-ci n'est pas bonne ou mauvaise en soi, mais selon l'usage que l'on en fait. De ce point de vue, des solutions techniques peuvent ainsi être adoptées pour arriver à une "société durable ". L'exemple typique de cette pensée réside dans "l'écologie industrielle ", cette manière de considérer l'économie non comme un rapport social, mais comme un système technique d'entrants et d'extrants qu'il s'agit d'optimiser.

Ces trois exemples donnent une idée des types d'oppositions que l'on peut déceler entre ces courants. En forçant les traits de l'une et l'autre approche, on peut définir deux idéaux-types recouvrant les principales oppositions. Dans les faits, aucun acteur ne peut être entièrement rattaché à l'un ou l'autre pôle, chacun se positionnant quelque part entre les deux, mais cette distinction a un intérêt analytique (tableau 1). 
Tableau 1. Les types-idéaux de l'« écologie politique » et du « développement durable ».

\begin{tabular}{|l|l|}
\hline « Ecologie politique » & « Développement durable » \\
\hline Autonomie & Survie \\
\hline Présent & Futur (générations futures) \\
\hline Individu & Collectif/ espèce humaine \\
\hline Citoyens & Experts \\
\hline Gauche/extrême-gauche & Ni-droite, ni-gauche/ au-delà des clivages \\
\hline Société civile & Etat \\
\hline Démocratie directe & Démocratie participative / élite éclairée \\
\hline Auto-limitations & Limitations (hétéro-limitations) \\
\hline Choix & Nécessité \\
\hline Critique de la technique/ science & Technique/science \\
\hline Conflit & Harmonie/ stratégies « gagnant-gagnant » \\
\hline $\begin{array}{l}\text { Décentralisation/fédéralisme européen } \\
\text { /régionalisme }\end{array}$ & $\begin{array}{l}\text { Planification / intergouvernemental/ } \\
\text { gouvernance mondiale }\end{array}$ \\
\hline Révolution/ réforme radicale & Réforme modérée/ conservatisme \\
\hline Rupture & Continuité \\
\hline $\begin{array}{l}\text { Changement du mode de production/ } \\
\text { décroissance/convivialité }\end{array}$ & $\begin{array}{l}\text { Réorientation écologique/ écologie } \\
\text { industrielle/ «nouvelle ère de croissance » }\end{array}$ \\
\hline
\end{tabular}

Retenons de cette distinction, trop brièvement esquissée, que le développement durable se situe dans un entre-deux. Il est pris entre l'idéal écologique radical, tout en restant situé dans le cadre de la société industrielle productiviste. Empruntant à l'un et à l'autre, il ne se donne pas les moyens de dépasser leurs contradictions en prétendant les intégrer. Il apparait dès lors comme une tentative non-dialectique de concilier les contraires ; que ces contraires soient les pôles qu'il définit (environnement, économie, social) ou les contradictions internes à chacun de ces pôles. Comme si le développement économique (qu'il s'agirait de concilier dans une stratégie «doublement gagnante» avec l'environnement) était un processus technique et non un lieu conflictuel tissé de rapports de productions; comme si le social pouvait être défini comme un (un seul) intérêt particulier à concilier avec l'économie ou l'environnement.

\section{Démocratie et développement durable : un couple incertain}

De cette origine conceptuelle, on peut déduire le rapport particulier du développement durable à la démocratie, situé entre l'imaginaire radical de la démocratie directe et la pratique du gouvernement représentatif. Si les doctrines qui ont précédées le développement durable pouvaient se montrer franchement hostiles à la démocratie (en particulier la philosophie de Hans Jonas; par exemple Jonas, 2000, pp. 57-58), celui-ci entretient un rapport plus méfiant que défiant à celle-ci (OCDE, 2002, pp. 33 et 37). Dans la lignée de la "gouvernance» prônée par les institutions internationales, le développement durable valorise le concept de " démocratie participative », qui emprunte à la démocratie directe son poids symbolique, mais reste enchâssé dans le cadre du système représentatif, comme nous le verrons par la suite.

Le rapport Brundtland est assez laconique à cet égard, puisqu'il ne mentionne qu'une fois cette idée, et encore de manière très générale (Brundtland, 1987, p. 65). Pour l'ONU, la participation consiste à permettre aux citoyens concernés d'avoir accès à l'information 
environnementale, comme le demande le dixième principe de la déclaration de Rio (ONU, 1999, p. 10). C'est cette politique de participation que prône l'OCDE en encourageant à «améliorer la transparence et la participation du public à tous les niveaux des gouvernements :

- En permettant la participation effective des entreprises, des travailleurs, des consommateurs et des organisations non-gouvernementales aux débats sur les modes de production et de consommation, de manière à faciliter la transition vers le développement durable ;

- En donnant au public un accès à l'information et à des moyens efficaces de faire entendre sa voix (par exemple procédures judiciaires)» (OCDE, 2001, p. 29). Ainsi, du point de vue du développement durable, la "participation " a une valeur purement instrumentale, qui se décline sur deux plans : la consultation et le consensus.

\section{La participation-consultation}

De ce point de vue, la participation permettrait une meilleure circulation de l'information dans le système de pilotage politique. Les gouvernants prendraient en effet des mauvaises décisions à cause d'un déficit d'information en provenance de la base. Les procédures participatives, au contraire, permettraient d'institutionnaliser une manière de faire remonter les savoirs et les désirs locaux, afin que le pouvoir politique puisse décider en connaissance de cause. Il ne s'agit donc pas, par la participation, de créer un espace de débat politique, mais plutôt d'instaurer une modalité "technique " de l'information gouvernementale. Les discussions doivent alors être sérieusement encadrées (OCDE, 2001, p. 65). Ceci ne signifie évidemment pas que la consultation soit une mauvaise chose ; bien des « horreurs » urbanistiques et architecturales peuvent être évitées par ce biais.

\section{La participation-consensus}

Par ailleurs, la participation serait un moyen de faire comprendre et accepter les décisions politiques à l'ensemble des citoyens. Il s'agit de leur soumettre une proposition, ou un projet, et de les faire débattre à ce sujet. En leur donnant l'occasion de participer, de discuter, de débattre des projets, les citoyens seraient ainsi amenés à comprendre les motivations fondant ces politiques et à les accepter plus facilement. Il ne s'agit nullement d'organiser un débat sur le bien-fondé des projets présentés, mais de les faire comprendre et accepter parce que nécessaires. Comme le dit l'OCDE (2001, p. 65) : « La transparence des activités des pouvoirs publics est nécessaire pour offrir des possibilités de participation active des citoyens au débat sur le développement durable et forger un consensus sur les réformes nécessaires. " La participation serait donc un moyen de gouvernement qui permettrait d'éviter des blocages ou des oppositions trop nombreuses, en incorporant les désirs et les demandes des citoyens.

\section{L'exemple des Agendas 21 locaux}

Les deux modalités de procédures participatives du développement durable sont particulièrement importantes dans le cas de l'application des "agendas 21 locaux » à l'échelle urbaine. L' "agenda 21 « est le programme d'action de l'ONU pour la planète, décidé à Rio. Il se décline en programmes locaux, au niveau des régions, des municipalités ou des quartiers sous le nom d'« Agenda 21 locaux«. Suivant la «charte d'Aalborg", 
signée en 1994 , de nombreuses villes européennes participent à ce développement et incluent désormais des procédures participatives, notamment en matière d'aménagement local pour le développement durable. Il s'agit en fait d'adapter le vieux principe de « mise à l'enquête » en matière d'urbanisme au goût du développement durable, en l'élargissant et le modernisant. La « participation-consultation » en est un facteur essentiel ${ }^{2}$.

20 La participation telle qu'entendue par la théorie du développement durable repose donc sur l'idée qu'il faut élargir l'accès à l'information pour des individus et des groupes particuliers. En retour, ces individus et ces groupes constituent une source d'information privilégiée pour le pouvoir central. Les individus, en participant, prennent conscience des enjeux du développement durable et acceptent ses «réformes nécessaires». Ceci peut être vu comme une avancée par rapport aux conceptions hiérarchiques (top-down) de l'urbanisme des années 1960, néanmoins cette participation ne constitue pas un accès formel élargi à la décision politique.

\section{Démocratiser la démocratie : l'échec participatif}

21 Cette acception de la "démocratie participative " permet-elle une démocratisation de l'action publique? Après avoir exposé le contenu de cette forme politique, selon le développement durable, nous devons la réinscrire dans son opposition à la démocratie directe et au gouvernement représentatif. Elle peut sembler s'opposer ou s'adjoindre au gouvernement représentatif, alors qu'elle ne fait que s'inscrire dans ses cadres. Il apparaîtra alors que cette idée de «l'entre-deux » ne peut pas apporter un supplément de démocratie.

\section{Gouvernement représentatif et division du travail}

Le gouvernement représentatif est la conséquence logique d'une société industrielle productiviste, qui a besoin de se spécialiser afin de croître. Il s'agit de l'application du principe de division du travail à la politique. Le grand théoricien du libéralisme postrévolutionnaire, Benjamin Constant, affirme que : «Le système représentatif n'est autre chose qu'une organisation à l'aide de laquelle une nation se décharge sur quelques individus de ce qu'elle ne peut ou ne veut pas faire elle-même. Les individus pauvres font eux-mêmes leurs affaires ; les hommes riches prennent des intendants. » (Constant, 1997 [1819], p. 615).

L'avènement du gouvernement représentatif ne doit pas se comprendre uniquement comme la résultante d'un mode de production particulier, il est également là pour protéger les élites contre les assauts du peuple, comme l'a admirablement montré Bernard Manin (1996). Néanmoins, pour ce qui nous intéresse, conservons à l'esprit le lien entre système économique productiviste et nécessité de spécialiser l'activité politique, au travers de représentants dans sa forme la plus démocratique.

L'écologie politique, anti-productiviste, refuse la spécialisation à outrance des sociétés industrielles. Fortement marquée par les conceptions antiques de la politique (démocratie athénienne, république...) elle pense que la politique n'est pas un domaine parmi d'autres de l'activité humaine, mais qu'elle est l'activité de tous. Il ne saurait donc exister une classe politique spécialisée qui se chargerait, au nom des autres, des affaires politiques. L'écologie politique met donc en avant une conception directe de la 
démocratie, qui se décline notamment sur le thème de l'autonomie individuelle et de l'autogestion (Castoriadis, 2005 [1992]).

Le développement durable se trouve à nouveau pris dans un entre-deux, tiraillé entre l'imaginaire radical de l'écologie politique et son insertion dans les cadres de la société industrielle de croissance. Il développe l'idée de « démocratie participative » qui, tout en empruntant à l'imaginaire de la démocratie directe, reste formellement enchâssée dans le système représentatif. A cet égard, la démocratie participative ne peut pas apporter un surcroît de démocratie.

\section{Les types idéaux de la démocratie ${ }^{3}$}

Afin de mieux comprendre pourquoi et de cerner les différences principales entre ces conceptions, nous proposons un schéma idéal-typique des trois conceptions, de leur rapport à la démocratie et surtout de trois critères politiques fondamentaux dans la théorie politique (tableau 2). Il s'agit de localiser le lieu du débat politique. Puis le lieu ultime de décision politique, répondant à la question classique "qui, en dernière instance, a le pouvoir dans ce système? ». Enfin, il s'agit d'identifier le mécanisme de responsabilité à l'oeuvre dans chaque système.

Tableau 2. Types idéaux de démocratie.

\begin{tabular}{|l|l|l|l|l|l|}
\hline \multicolumn{2}{|l|}{ Conception économico-politique } & $\begin{array}{l}\text { Type de } \\
\text { démocratie }\end{array}$ & Débat & Décision & Responsabilité \\
\hline $\begin{array}{l}\text { Contestation } \\
\text { écologiste }\end{array}$ & $\begin{array}{l}\text { Ecologie } \\
\text { politique }\end{array}$ & $\begin{array}{l}\text { Démocratie } \\
\text { directe }\end{array}$ & $\begin{array}{l}\text { Tous/ } \\
\text { n'importe qui }\end{array}$ & $\begin{array}{l}\text { Tous/n'importe } \\
\text { qui }\end{array}$ & $\begin{array}{l}\text { Immédiate } \\
\text { (a prioni) }\end{array}$ \\
\cline { 2 - 5 } & $\begin{array}{l}\text { Développement } \\
\text { durable }\end{array}$ & $\begin{array}{l}\text { Démocratie } \\
\text { «participative » }\end{array}$ & $\begin{array}{l}\text { Tous/ } \\
\text { n'importe qui }\end{array}$ & $\begin{array}{l}\text { Représentants/ } \\
\text { gouvernants }\end{array}$ & Diluée/ illisible \\
\hline $\begin{array}{l}\text { Société industrielle de croissance, } \\
\text { libéralisme }\end{array}$ & $\begin{array}{l}\text { Démocratie } \\
\text { représentative }\end{array}$ & Représentants & Représentants & $\begin{array}{l}\text { Réélection } \\
\text { (a posterioni) }\end{array}$ \\
\hline
\end{tabular}

De manière très classique, la démocratie représentative se caractérise par le fait que, si tous les citoyens peuvent formellement participer au choix de leurs élites - les représentants -, ce sont ces derniers qui ont le pouvoir de débattre et de décider. Néanmoins ces représentants sont responsables (accountable), de leurs actions. Les citoyens exercent un contrôle a posteriori de leurs dirigeants en choisissant de les réélire ou non, ce qui produit un effet réel sur la politique menée par les représentants durant leur mandat (Manin, 1996, p. 228). Cette responsabilité est la contrepartie de la délégation de pouvoir dont ils bénéficient.

La démocratie directe prônée par l'écologie politique - de même que l'autogestion suppose que tout un chacun prend une égale part au débat et à la décision politique. Il n'existe pas une classe séparée de gouvernants, mais un peuple qui s'auto-gouverne. La responsabilité est donc immédiate, c'est à dire que les individus subissent directement les conséquences de leurs choix . La démocratie athénienne, qui inspire en fait cette conception, faisait reposer l'idée démocratique non sur la représentation, mais sur la rotation des charges. A cet égard, le seul mécanisme démocratique pouvait être le tirage au sort et non l'élection, principe aristocratique par excellence (Finley, 1985; Manin, 1996 ; Rancière, 2005).

La démocratie participative tente de concilier ces deux principes opposés. Elle suppose l'existence de gouvernants (représentants ou autres), mais elle leur adjoint des enceintes 
où tout un chacun, formellement, peut "participer ", c'est-à-dire donner son avis. Elle mobilise ainsi l'imaginaire "délibératif » habermassien de la démocratie et les effets positifs qui lui sont associés, tout en restant formellement dans les cadres décisionnels du gouvernement représentatif. Comme nous l'avons vu, les procédures « participatives » du développement durable ressortent essentiellement de deux objectifs : l'information des gouvernants et l'adhésion des citoyens; la consultation et le consensus.

Cette "participation » suppose donc qu'il existe deux enceintes séparées, entre le débat et la décision. Alors que le modèle direct et le modèle représentatif font coïncider ces deux lieux, le modèle participatif les sépare. D'une part se trouvent des enceintes formellement ouvertes à tous pour participer. Les citoyens peuvent y venir librement et donner leur avis, mais rien ne garantit qu'ils seront écoutés, car, d'autre part, existe une enceinte légitime de décision, qui ne compte que les gouvernants. Certes, il peut exister de fortes incitations pour que les gouvernants suivent les discussions et avis forgés au sein des institutions participatives, mais il n'y a là aucune obligation. Les procédures "participatives» ne peuvent donc formellement accroitre le pouvoir d'un citoyen indéterminé de décider politiquement.

31 Néanmoins, les gouvernants peuvent utiliser l'existence d'enceintes "participatives" afin de diluer ou esquiver leur responsabilité. Ils peuvent ainsi jouer gagnants sur deux tableaux : avoir le pouvoir effectif de décision, et rejeter la responsabilité d'une décision sur les citoyens qui ont "participé » à celle-ci. Il s'agit là d'une pratique bien connue en matière d'aménagement. Ainsi, la responsabilité est diluée dans le modèle " participatif ». D'un point de vue normatif et formel, la démocratie participative, au sens du développement durable, n'apporte donc pas plus de démocratie, au sens de participation effective à la prise de décision politique, que le gouvernement représentatif.

\section{La délibération contrainte}

Par ailleurs, ces institutions ou procédures participatives vont à l'encontre d'un autre trait fondamental de la démocratie, qui est l'indétermination a priori du débat. Que ce soit dans l'agora athénienne ou dans les parlements représentatifs, les participants ont la maîtrise des sujets dont ils veulent débattre et ne sont pas soumis à une loi extérieure. Claude Lefort fait même de l'« indétermination » la caractéristique fondamentale de la démocratie (Lefort, 1986). Les procédures "participatives « au contraire supposent la passivité des citoyens, auxquels on pose une question précise. La "participation" consiste à s'exprimer sur un plan d'urbanisme, sur un aménagement local, sur une question éthico-technologique, etc. Il s'agit donc d'une procédure visant à faire débattre un groupe de citoyens sur une question déterminée. Le fait de poser cette question, la décision d'ouvrir une procédure participative et la décision finale sur cette question restent du ressort des gouvernants, ce qui n'invalide pas l'intérêt instrumental que peuvent avoir ces pratiques, mais empêche de les créditer de « démocratisation ».

\section{Le développement durable, menue monnaie des alternatives déchues}

La démocratie participative n'apporte donc pas plus de démocratie que les formes qui lui préexistent. Elle est symboliquement dans l'entre-deux (entre démocratie directe et 
démocratie représentative), mais d'un point de vue formel reste enchâssée dans le gouvernement représentatif. Comme le note Jacques Rancière (2004, p. 110) : "Je me demande pourtant si cette notion qu'on donne comme solution aux problèmes de la démocratie n'est pas plutôt une solution aux problèmes de sa critique, la menue monnaie des grandes alternatives déchues. »

Or cette menue monnaie se comprend mieux encore si on la considère en lien avec le développement durable, lui aussi le produit des grandes alternatives déchues. Tiraillé entre l'imaginaire radical de l'écologie politique, et les contraintes matérielles de la société de croissance industrielle, le développement durable ne tente pas le dépassement dialectique de leurs contradictions, mais les juxtapose sur un mode idéaliste. Il est une tentative non-dialectique de concilier les contraires - ce que Lopreno (1989) nomme un «concept kitsch» - comme le montrent les expressions telles que «stratégies doublement ou triplement gagnantes » ou l'oxymoron de « l'écologie industrielle ».

A cet égard, le succès phénoménal du développement durable, repris en coeur par tous les acteurs sociaux et économiques, pourrait témoigner moins de sa pertinence que de sa profonde innocuité. Les raisons de l'émergence d'un tel concept sur la scène politique comme sur la scène académique mériteraient d'être investiguées. Il pourrait alors être compris comme l'alternative d'un monde sans alternative.

\section{BIBLIOGRAPHIE}

ALPHANDERY P., BITOUN P., DUPONT Y. (1991), L'équivoque écologique, Paris, La Découverte.

BACQUE M.-H., REY H., SINTOMER Y. (2005), Gestion de proximité et démocratie participative, Une perspective comparative, Paris, La Découverte.

BEVORT A. (2002), Pour une démocratie participative, Paris, Presses de Sciences-Po.

BOURG D. (1996), Les scénarios de l'écologie, Paris, Hachette.

BOURG D., BOY D. (2005), Conférences de citoyens, mode d'emploi : les enjeux de la démocratie participative, Paris, Descartes et Cie/Charles Léopold Mayer.

BRUNEL S. (2004), Le développement durable, Paris, PUF.

CASTORIADIS C., COHN-BENDIT D. (1981), De l'écologie à l'autonomie, Paris, Seuil.

CASTORIADIS C. (2005 [1986]), « Les enjeux actuels de la démocratie« , in Une société à la dérive, pp. 155-160, Paris, Seuil.

CASTORIADIS C. (2005 [1992]), «L'écologie contre les marchands », Une société à la dérive, pp. 237-239, Paris, Seuil.

CONSTANT B. (1997 [1819]), « De la liberté des anciens comparée à celle des modernes », in Ecrits politiques, Paris, Gallimard, pp. 589-619.

FINLEY M. (1985 [1983]), L'invention de la politique, Paris, Flammarion.

GORZ A. (1978), Ecologie et politique, Paris, Seuil. 
ILLICH I. (1973), La convivialité, Paris, Seuil.

JAKUBEC J. (2004), Le développement durable. Un bilan multisectoriel provisoire, Genève et Paris, Georgs éditeurs.

JONAS H. (2000 [1993]), Une éthique pour la nature, Paris, Desclée de Brouwer.

LEFORT C. (1986), « La question de la démocratie », in Essais sur le politique, XIX ${ }^{e}-X X^{e}$ siècle, Paris, Seuil, pp. 17-32.

LOPRENO D. (1989), Le concept de post-industrialisme, Essai critique sur la transformation du tissu industriel genevois, Genève, Le Concept Moderne.

MANIN B. (1996), Principes du gouvernement représentatif, Paris, Flammarion.

OCDE (2002), La gouvernance pour le développement durable, Etude de cinq pays de l'OCDE, Paris, OCDE.

OCDE (2001), Développement durable, Quelles politiques?, Paris, OCDE.

ONU (1999 [1993]), Earth Summit Agenda 21, The United Nations Programme of Action From Rio, Genève, United Nations Publications.

RANCIERE J. (2004), Aux bords du politique, Paris, Gallimard.

RANCIERE J. (2005), La haine de la démocratie, Paris, La fabrique.

RUI S. (2004), La démocratie en débat, Les citoyens face à l'action publique, Paris, Armand Colin.

SMOUTS M.-C. et al. (2005), Le développement durable : les termes du débat, Paris, Armand Colin.

THE WORLD COMMISSION ON ENVIRONMENT AND DEVELOPMENT (1987), Our Common Future, Oxford, New York, Oxford University Press (« rapport BRUNDTLAND »).

VERNANT J.-P. (1962), Les origines de la pensée grecque, Paris, PUF.

VIVIEN F.-D. (1994), Economie et écologie, Paris, La Découverte.

ZACCAI E. (2002), Le développement durable, Dynamique et constitution d'un projet, Bruxelles, PIEPeter Lang.

\section{NOTES}

1. Louis Schweitzer, PDG de Renault, Enjeux Les Echos, mensuel, Paris, décembre 2004.

2. Voir le point I-13 de cette Charte des villes européennes pour la durabilité - Charte d'Aalborg. Disponible sur: http://www.ecologie.gouv.fr/IMG/agenda21/textes/aalborg.htm [référence du 29 mai 2005].

3. A proprement parler, on ne saurait qualifier le gouvernement représentatif de «démocratie» (Manin, 1996, pp. 305-308). Néanmoins, par simplification nous utilisons ce raccourci. 


\section{RÉSUMÉS}

Cet article vise à comprendre le lien entre développement durable et démocratie participative, d'un point de vue critique. Après avoir montré que le développement durable doit être compris en opposition à l'écologie politique, il interroge la conception de la démocratie que celui-ci développe. A la suite d'un raisonnement formel, il conclut que la démocratie participative, telle qu'entendue par le développement durable, n'apporte pas un supplément de démocratie. Cette situation doit être comprise comme la résultante de la tentative non-dialectique de concilier les contraires qu'est le développement durable.

This paper seeks to understand the link between sustainable development and participative democracy, from a critical point of view. After having shown that sustainable development has to be understood in opposition to political ecology, it questions its conception of democracy. Following a formal reasoning, it concludes that participative democracy, as understood by sustainable development, does not provide a democratization. This situation has to be understood as a result of the ideology of sustainable development: a non-dialectical attempt to reconcile contraries.

\section{INDEX}

Mots-clés : développement durable, démocratie participative, écologie politique, théorie politique, démocratie urbaine

Keywords : sustainable development, participative democracy, political ecology, political theory, urban democracy

\section{AUTEUR}

\section{ROMAIN FELLI}

Université de Lausanne, romain.felli@unil.ch 